\title{
Acute hemorrhagic leukoencephalitis in a COVID-19 patient-a case report with literature review
}

\author{
Baskaran Varadan ${ }^{1} \cdot$ Abhinaya Shankar $^{1} \cdot$ Akila Rajakumar $^{2}$ - Shubha Subramanian ${ }^{3}$ - A. C. Sathya ${ }^{4}$. \\ Abdul Rahman Hakeem ${ }^{5}$. Srinivasan Kalyanasundaram ${ }^{1}$
}

Received: 16 December 2020 / Accepted: 3 February 2021 / Published online: 11 February 2021

(C) The Author(s), under exclusive licence to Springer-Verlag GmbH, DE part of Springer Nature 2021

\begin{abstract}
Purpose Acute hemorrhagic leukoencephalitis (AHLE) is a rare and severe form of acute disseminated encephalomyelitis (ADEM). Only a few reports of AHLE in coronavirus disease 2019 (COVID-19) patients have been described to date. We report a case of COVID-19-related AHLE along with a literature review describing salient clinical and imaging characteristics. Methods A literature search was performed on Medline (2020-present), PubMed, Cochrane Library, CINAHL, and Google scholar on 28 January 2021 for all articles published using MeSH terms "COVID-19" or "SARS-CoV-2" with "Acute hemorrhagic leukoencephalitis" or "Acute hemorrhagic encephalitis." Relevant case reports and case series describing clinical and imaging features of AHLE associated with SARS-CoV-2 infection were included, data compiled, and critically reviewed.

Results Acute onset encephalopathy and rapidly deteriorating neurological status is the common clinical presentation in AHLE. CSF analysis reveals elevated proteins and lymphocytic pleocytosis. Typical neuroimaging features include multifocal, variablesized, poorly defined cerebral white matter lesions with cortical sparing. Involvement of the brainstem, cerebellar peduncles, and deep grey matter can also occur, although rarely. Lesions are hyperintense on T2-weighted (T2W) and fluid-attenuated inversion recovery (FLAIR) images, hypointense on T1W images, and show microhemorrhages, variable diffusion restriction, and postcontrast enhancement. Extensive microhemorrhages, brainstem involvement, and gross hemorrhage often portend a poor prognosis.

Conclusion Heightened awareness about the clinical and imaging presentation of COVID-19-related AHLE can positively alter the outcome in a select few by enabling early diagnosis and aggressive management.
\end{abstract}

Keywords COVID-19 - Acute hemorrhagic leukoencephalitis (AHLE) • Acute disseminated encephalomyelitis (ADEM) · Computed tomography $(\mathrm{CT}) \cdot$ Magnetic resonance imaging $(\mathrm{MRI})$

Srinivasan Kalyanasundaram

drksvasan@gmail.com

1 Department of Radiology, Dr. Rela Institute and Medical Centre, Chromepet, Chennai, India

2 Liver Intensive Care and Anaesthesia, Clinical Lead, Critical Care Services, Dr. Rela Institute and Medical Centre, Chromepet, Chennai, India

3 Department of Neurology, Dr. Rela Institute and Medical Centre, Chromepet, Chennai, India

4 Intensive Care, Department of Anaesthesia and ICU, Dr. Rela Institute and Medical Centre, Chromepet, Chennai, India

5 HPB Surgery and Liver Transplantation, Institute of Liver Diseases \& Transplantation, Dr. Rela Institute \& Medical Centre, Chromepet, Chennai, India

\section{Introduction}

Acute hemorrhagic leukoencephalitis (AHLE), a subtype of acute disseminated encephalomyelitis (ADEM), is a rare and severe form of post-infective demyelination. Viral infections, including those caused by coronaviruses, are common precedents. The ongoing coronavirus disease 2019 (COVID-19) pandemic caused by severe acute respiratory syndrome coronavirus 2 (SARS-CoV-2) has resulted in morbidity and mortality on an unprecedented scale. Of the different neurological complications encountered in COVID-19 patients, a few can be fatal, and AHLE is one of them. We share our experience with AHLE in a COVID-19 patient, who had a fulminant clinical course, presented with florid imaging findings, unfortunately culminating in a fatal outcome. A brief literature 
review is provided highlighting the salient clinical and imaging features of COVID-19-related AHLE.

\section{Case presentation}

A 46-year-old gentleman with a background of alcoholrelated chronic liver disease, presented with fever and breathlessness for 3 days. His liver disease was stable on medical management. COVID-19 infection was suspected and confirmed with a nasal swab reverse transcriptase-polymerase chain reaction (RT-PCR). Computed tomography (CT) of the chest revealed subpleural ground-glass opacities and consolidation in bilateral lower lobes, typical for COVID-19 pneumonia. In view of his stable observations and inflammatory markers, the patient was managed conservatively and discharged 4 days later with advice on home quarantine.

Five weeks following discharge, he presented to the emergency department with main complaints of headache and altered mental status. At admission, he was tachycardic (pulse rate $100 / \mathrm{min}$ ), with normal blood pressure and was hypoxic with oxygen saturation of $93 \%$ on room air. His admission Glasgow Coma Score (GCS) was 11/15 (E3V3M5). Neurological examination revealed a complete loss of power in the left upper limb (0/5) and reduced power in the left lower limb (3/5), with signs of left facial nerve palsy. Deep tendon and brainstem reflexes were intact. A provisional diagnosis of acute cerebrovascular accident was made. His admission Model for End-Stage Liver Disease (MELD) score was 20.

Initial imaging evaluation with non-contrast $\mathrm{CT}$ brain revealed multifocal non hemorrhagic white matter lesions in both cerebral hemispheres and the brainstem (Fig. 1). Magnetic resonance imaging (MRI) performed subsequently confirmed white matter lesions in bilateral frontal, parietal lobes, left thalamus, left cerebral peduncle, and medulla. These lesions were hyperintense on T2-weighted (T2W) and fluid-attenuated inversion recovery (FLAIR) images and hypointense on T1-weighted (T1W) images. Internal areas of diffusion restriction and irregular patchy areas of rim enhancement were noted within most of the lesions. Left parietal periventricular white matter lesion was reaching the ventricular atrium with subjacent faint subependymal enhancement. Few microbleeds were seen within this lesion (Fig. 2). Elevated lactate was noted in MR spectroscopy. MRI brain angiogram, venogram, and spinal cord evaluation were unremarkable.

Due to the worsening clinical and neurological status, the patient was intubated and started on intravenous methylprednisolone $1 \mathrm{~g}$, based on a working diagnosis of COVID-19related encephalitis, given the temporal presentation following a respiratory infection. After ruling out papilledema, a lumbar puncture was done. Cerebrospinal fluid (CSF) showed lymphocytic pleocytosis with increased protein. Gram staining of
CSF was negative for bacteria and acid-fast bacilli. No demonstrable growth was noted in bacterial and fungal cultures. Cytology revealed no malignant cells. Inflammatory markers including D-dimer and ferritin were significantly elevated. Electroencephalogram (EEG) revealed no epileptiform discharges.

Aggressive antiedema measures were initiated. Despite 5 days of steroids, the patient's clinical and neurological status continued to deteriorate. A repeat MRI to assess disease evolution was performed, which revealed progression in number and size of the cerebral white matter and brainstem lesions, with florid intralesional hemorrhage (Fig. 3). Worsening edema with mass effect was noted causing bilateral uncal and central descending transtentorial herniation. Secondary vascular compression and consequent acute infarcts in bilateral posterior cerebral artery territories were also seen.

A final diagnosis of COVID-19-related AHLE was made, considering the rapid clinical deterioration, progression of imaging features, and CSF picture. Antiedema measures were continued, and decompressive craniotomy was planned. However, patient deteriorated rapidly and died on the same day.

\section{Methods}

An electronic search of Medline via PubMed (2020-present), Cochrane Library, CINAHL, and Google scholar was conducted independently by authors (BV and AS) on 28 January 2021. All the database searches were performed to identify studies reporting on "COVID-19" or "SARS-CoV2" with "Acute hemorrhagic leukoencephalitis" or "Acute hemorrhagic encephalitis." The search strategy was based on the following combination of terms: (coronavirus disease OR coronavirus OR COVID OR SARS-CoV OR SARS-CoV-2) AND (acute hemorrhagic OR acute haemorrhagic) AND (encephalitis OR leucoencephalitis OR leukoencephalitis). Out of 78 articles written in English, published since 1 January 2020, 6 articles reporting clinical and neuroimaging manifestations of AHLE were selected for review. Data from these reports was compiled (Table 1) and critically reviewed.

\section{Discussion}

AHLE, a monophasic demyelinating disease and a hyperacute severe form of ADEM, is a rare clinical entity with a poor prognosis. There is antecedent history of viral or bacterial infection in $50-75 \%$ of patients, with most cases following an upper respiratory tract infection [1]. ADEM is not uncommon during childhood, with hospitalization estimated at $\sim 0.5$ per 100,000 patients $[2,3]$. A much lower incidence has been reported in adults [1]. In comparison to other presentations of 
Fig. 1 a CT brain axial sections showing focal hypodensities involving the left hemimedulla. b Hypodensities involving the right centrum semiovale, frontal, and left parieto-occipital white matter without hemorrhage. $\mathbf{c}$ and $\mathbf{d}$

Sagittal and coronal images demonstrating the left parietooccipital deep white matter lesion. No hemorrhage or mass effect

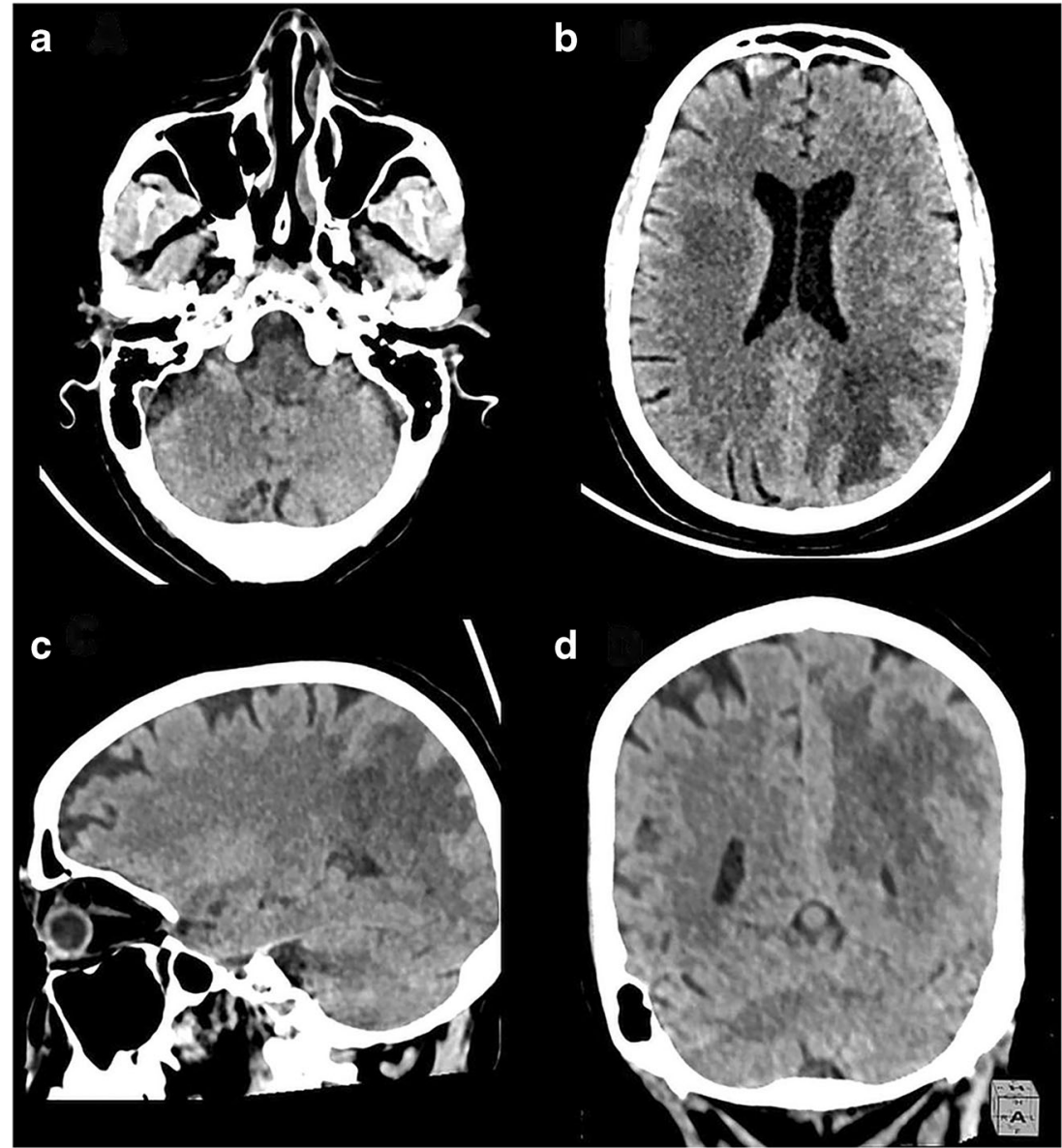

ADEM, AHLE is much rarer, and there are only a few case reports describing this entity [1]. Infective cause was not demonstrable in a few cases, and there may not be a linear relationship between the severity of neurological illness and antecedent chest symptoms $[1,4]$. Though neurological symptoms are common in COVID-19 patients, only a handful of COVID-19 associated AHLE have been reported to date [5-11].

Neurological manifestations in COVID-19 are considered secondary to direct viral cytopathic effect on neurons, immune-mediated inflammation, and development of intracranial cytokine storm $[9,12]$. Recent reports show significantly elevated levels of cytokines in patients with AHLE in comparison to those with ADEM and non-inflammatory neurological conditions [12]. Direct endothelial injury and/or secondary inflammation by SARS-CoV-2 is also considered to play a role, as suggested by a vasculitis-like presentation in some patients $[13,14]$. Pathological features in AHLE include perivascular inflammatory infiltrates, demyelination, necrotizing vasculitis, fibrinoid necrosis, and hemorrhages [15]. Limited neuropathological data in COVID-19 patients also shows similar features without a demonstrable infectious agent, which supports a possible para-infectious causative mechanism $[16,17]$.
Clinically, majority of AHLE patients are critically ill with acute onset encephalopathy and deteriorate rapidly within a few days. Almost half of the affected patients eventually succumb due to disease progression, and those that survive suffer from significant neurological sequelae [1]. In most cases, laboratory parameters reveal elevated levels of inflammatory markers such as C-reactive protein (CRP), D-dimer, serum ferritin, and procalcitonin. CSF analysis shows elevated protein, variable cell count, and lymphocytic pleocytosis [6-8]. Cultures are often negative, and viral RNA is rarely demonstrated in CSF; hence, RT-PCR for CSF was not done in our patient $[9,18,19]$.

Typical neuroimaging features of AHLE include multifocal, variable-sized (often more than $1 \mathrm{~cm}$ ) poorly defined white matter lesions involving both cerebral hemispheres, predominantly parietal and occipital lobes. Subcortical and deep white matter predominance with characteristic asymmetric distribution is seen. Although less frequent, there can be brainstem, cerebellar peduncle, and deep grey matter involvement $[5,7]$. The lesions are hyperintense on T2W and FLAIR images and hypointense on T1W images and show microhemorrhage-related blooming in susceptibilityweighted images (SWI). Diffusion and contrast enhancement characteristics are variable. Extensive brainstem involvement 


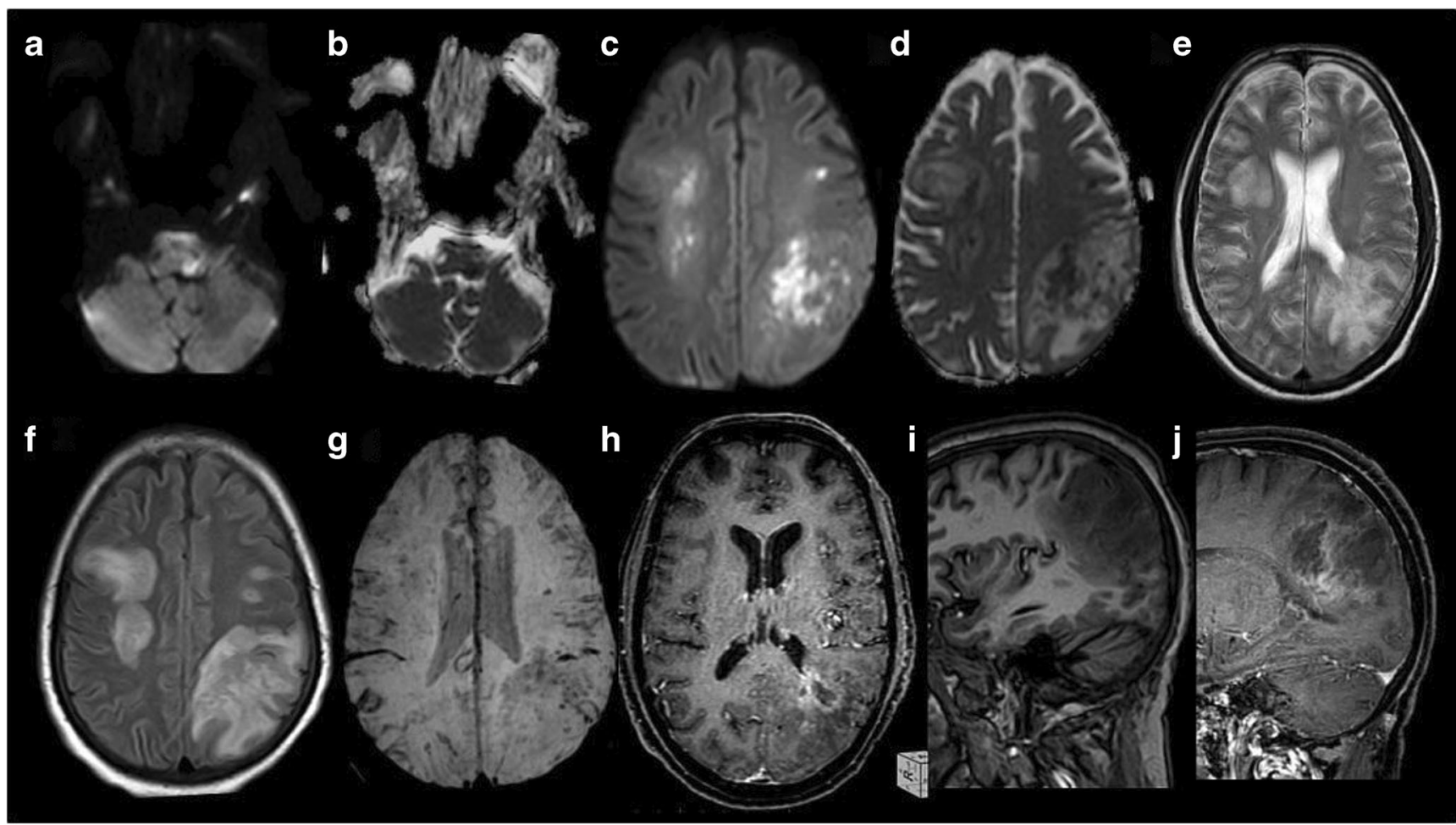

Fig. 2 MRI brain images - DWI images (a, c) and corresponding ADC maps $(\mathbf{b}, \mathbf{d})$ show diffusion restriction of lesions shown in CT with hyperintensity in T2WI (e) and FLAIR(f). Internal hemorrhage causing

and widespread microhemorrhages often portend a poor prognosis [19-21]. Unifocal pseudotumoral lesions involving the susceptibility changes in SWI images (g) seen. Pre contrast (i) and postcontrast $(\mathbf{h}, \mathbf{j})$ of lesions show patchy, rim enhancement with central nonenhancing component

corpus callosum and brainstem are rare presentations $[9,22$, 23].

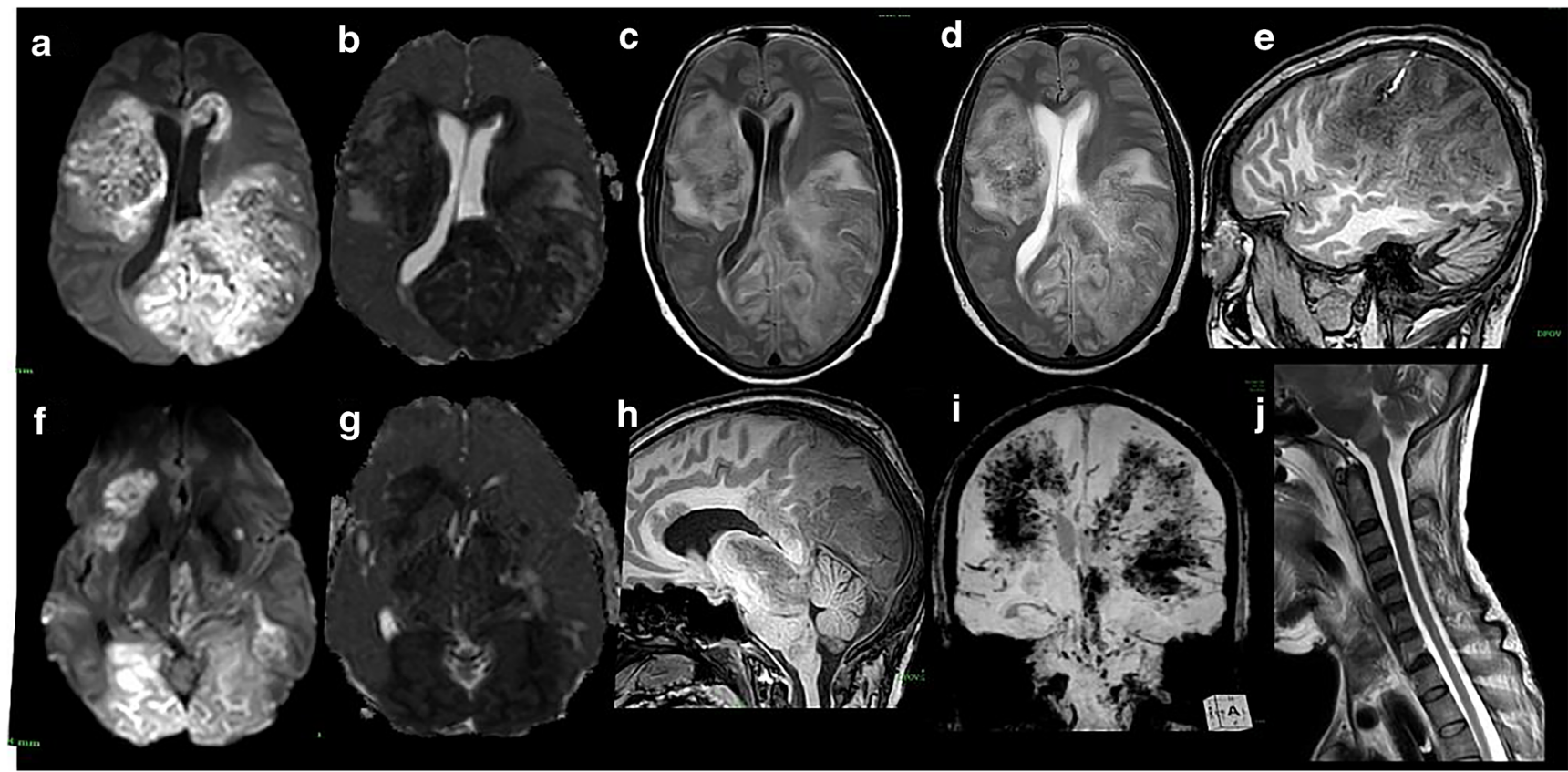

Fig. 3 Follow-up MRI revealed a significant interval increase in the size of lesions in the cerebral hemispheres (a to d) with new-onset left parietal subarachnoid hemorrhage (e). Interval increasing mass effect with associated uncal and descending transtentorial herniation (h) and acute infarcts in bilateral posterior cerebral artery territories (f, g). Florid intralesional and brainstem hemorrhage depicted (i). Sagittal T2W image of cervical spine shows no focal lesion in cervical spinal cord. T2 hyperintense medullary lesion (j). MR brain angiogram and venogram are normal. MR spectroscopy showed elevated lactate levels (not shown in images) 


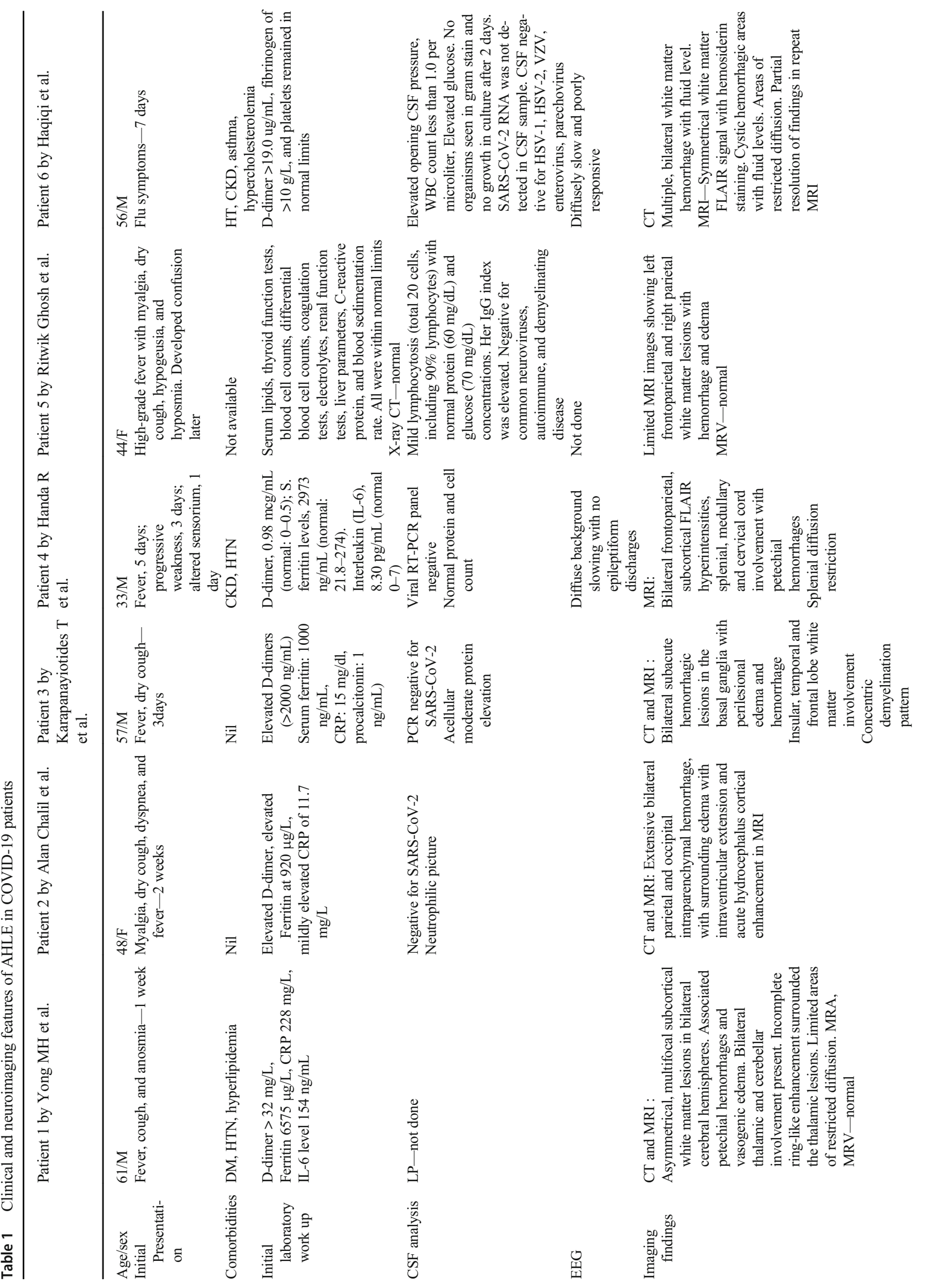




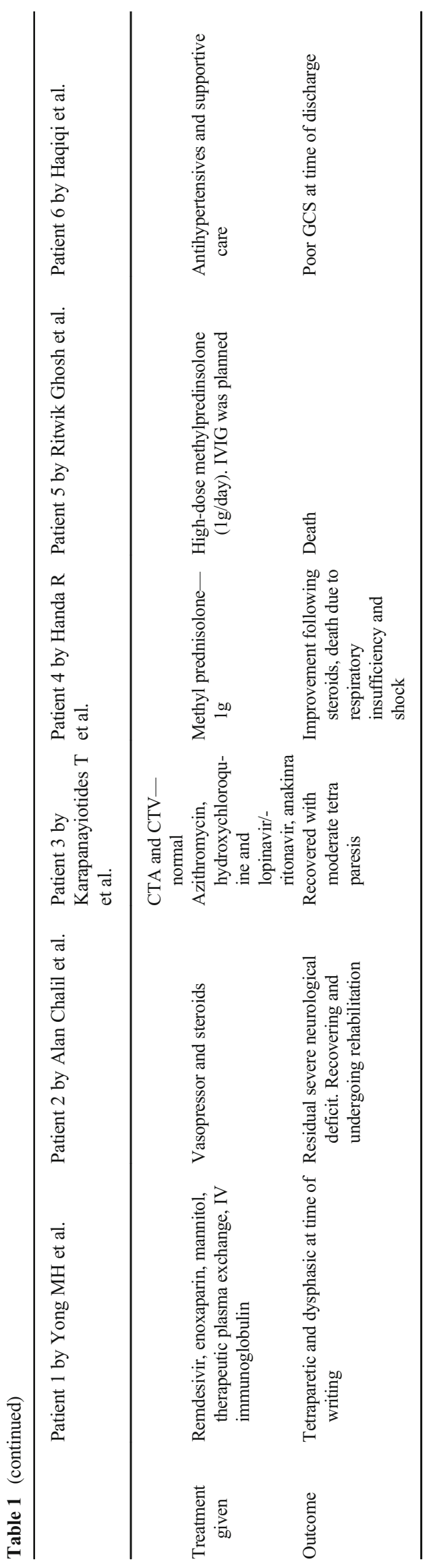

Microhemorrhages and leukoencephalopathy-related changes are however commonly seen in critically ill COVID-19 patients with or without clinical features of ADEM or AHLE. The presumed causal factors include direct infection, hypoxia, sepsis, PRES, and metabolic causes which are common in these patients [24]. However, the presence of white matter changes with florid micro or gross hemorrhage causing mass effect are imaging features favoring AHLE. Brainstem, cerebellar involvement, and widespread hemorrhage are features associated with a poor outcome [1].

In our patient, early imaging features showed minimal susceptibility changes with subtle subependymal enhancement which confounded the diagnosis requiring a wider differential including tumefactive demyelination and primary central nervous system (CNS) lymphoma. Follow-up MRI depicted rapid progression with florid parenchymal hemorrhages, which against this clinical background and CSF picture led us to make a final diagnosis of AHLE. The imaging features in our case parallel the findings demonstrated by Yong et al. and Chalil et al., albeit with severe edema, mass effect, and hemorrhagic component which could explain the fatal outcome $[5,6]$. Intraventricular hemorrhage described by Chalil et al. was not seen in our case [6]. The case described by Haqiqi et al. had similar widespread involvement, but lesions were symmetric without significant edema or mass effect. In this instance, the patient was hypertensive and on full dose of therapeutic anti-coagulation [11].

Handa et al. described bilateral frontoparietal white matter and cervical spinal cord signal changes without significant mass effect in COVID-19-associated AHLE. In addition, there was involvement of the splenium of corpus callosum [7]. Corpus callosum signal changes are common in ADEM and critically ill COVID-19 patients. Due to lack of SWI images, comparison of hemorrhagic severity with the rest of the AHLE cases and COVID-19 patients with splenial signal changes could not be made. Recovery of this patient along with atypical imaging pattern could suggest these features as a representation of a less severe variant of AHLE.

Karapanayiotides et al. reported a case of AHLE with concentric demyelinating lesions demonstrating a subacute hemorrhagic component and peripheral hemosiderin deposits [8]. Such an unusual appearance was not seen in our case neither has it been reported previously. In this case, there was predominant basal ganglia involvement with edema extending into the adjacent white matter, while most lesions in previously described reports were centered in the lobar white matter. Although hemorrhage was attributed to AHLE, the spontaneous clinical recovery and resolution of imaging findings remains uncharacteristic of usual AHLE patients. This case probably represents a unique variant of AHLE with a favorable outcome.

Pseudotumoral form of AHLE with corpus callosal involvement has been described in a non-COVID-19 and in 
one COVID-19 patient [9, 22]. Imaging in the non-COVID-19 patient showed florid splenial hemorrhage, and the diagnosis was histopathologically confirmed. However, in the COVID19 patient, AHLE was presumed based on imaging which showed callosal and frontoparietal grey-white matter junction microhemorrhages. Such punctate microbleeds (less than $3 \mathrm{~mm}$ ) in splenium and juxtacortical location are common nonspecific findings seen in critically ill COVID-19 patients [24]. The favorable outcome in the latter patient without immunosuppressive therapy is quite unusual for a case of AHLE. Diffuse white matter signal changes and widespread micro/ gross hemorrhages should be a prerequisite to entertain AHLE as a possible diagnosis, while a few microbleeds alone are more likely to be non-specific in COVID-19 setting. Ghosh et al. demonstrated hemorrhagic lesions in bilateral cerebral white matter which fits the description of AHLE [10].

Hypoxia, sepsis, and ischemic stroke are among the relatively more common entities that can cause acute neurological deterioration in COVID-19 patients. Other white matter diseases (ADEM, AHLE), necrotizing encephalitis (ANE), and vascular disorders (central venous sinus thrombosis (CVT); hemorrhagic posterior reversible encephalopathy syndrome (PRES)) have a much lower incidence but overlapping clinical manifestations. Hence a combination of imaging, CSF analysis, and histopathological evaluation help to narrow the differential.

ADEM, a milder form of post-infective encephalomyelitis, shows multiple, asymmetric, poorly marginated lesions which are relatively smaller in size, with less severe edema and mass effect and additional spinal cord involvement. Hemorrhage and enhancement are not a feature of these lesions [24, 25]. Basal ganglia are more commonly involved in ADEM than in AHLE. A fulminant variant of multiple sclerosis (MS) can be confused with AHLE, but it usually presents with callosal involvement and has characteristic CSF features. ANE has characteristic symmetric thalamic signal changes with additional involvement of brainstem, cerebral white matter, and cerebellum [26, 27]. Symmetric thalamic involvement is not seen in AHLE. Similar to AHLE, only rarely is the virus detected in CSF in ANE [28].

CVT is common in COVID-19 patients and can result in parenchymal hemorrhage. However, the clinical picture is different, and demonstrable thrombus in cortical veins and/ or dural venous sinuses establishes the correct diagnosis in most patients [29]. Hemorrhagic PRES also presents with parieto-occipital white matter involvement and hemorrhages. Few cases of hemorrhagic PRES in COVID-19 patients have been reported to date [30-32]. Cortical involvement can occur in PRES, and clinical presentation of elevated or fluctuating blood pressure is seen during the period of illness. Our index patient's blood pressure was within normal limits. Tocilizumab, a monoclonal antibody against interleukin-6, used on an experimental basis for treating cytokine storm in COVID-19 patients is also presumed to precipitate PRES [31]. It was not administered in our patient. Also, widespread hemorrhage as seen in our patient is not a typical feature of any of the abovementioned conditions.

To summarize, an imaging picture of multifocal, discrete, and confluent, variable-sized, poorly defined white matter lesions with significant hemorrhage, edema, and mass effect in a COVID-19 patient with rapid neurological deterioration should alert the treating physician towards a diagnosis of AHLE urging them to treat aggressively. High-dose IV steroids, often methylprednisolone, remain the first-line treatment [1]. Refractory cases can be treated with intravenous immunoglobulin (IVIG) and plasma exchange [1]. Based on a recent study on cytokines in COVID-19, tocilizumab along with broad-spectrum immunomodulatory drugs are suggested at the earliest suspicion of AHLE [12]. Treatment in COVID19-related AHLE is a relatively uncharted territory and bound to evolve with increasing experience and better understanding of the disease.

\section{Conclusion}

AHLE is a rare and often fatal neurological complication of COVID-19. Heightened clinical suspicion and early imaging identification of this entity can enable the clinicians to pursue more aggressive treatment options thereby reducing fatal outcomes.

Availability of data and responsibility The authors had full access to all the data and take responsibility for the integrity of the data.

Author contribution Dr. Baskaran Varadan and Dr. Abhinaya Shankar contributed to conception of design, data collection, review, analysis and interpretation of data, and drafting the article.

Dr. Akila Rajakumar, Dr. Shubha Subramanian, Dr. Sathya A.C, and Dr. Abdul Rahman Hakeem contributed to data review and drafting the article.

Dr. K. Srinivasan contributed to review, analysis and interpretation of data, drafting the article, and critical revision of the article.

\section{Declarations}

Conflict of interest The authors declare that they have no conflict of interest/competing interests.

Ethical approval All procedures performed in the studies involving human participants were in accordance with the ethical standards of the institutional and national research committee and with the 1964 Helsinki Declaration and its later amendments or comparable ethical standards.

Informed consent We have obtained the patient's informed consent and permission for the publishing of his information. 


\section{References}

1. Grzonka P, Scholz MC, De Marchis GM, Tisljar K, Rüegg S, Marsch S et al (2020) Acute hemorrhagic leukoencephalitis: a case and systematic review of the literature. Front Neurol 11:899. https:// doi.org/10.3389/fneur.2020.00899

2. Absoud M, Lim M, Chong WK, De Goede CG, Foster K, Gunny R et al (2013) Paediatric acquired demyelinating syndromes: incidence, clinical and magnetic resonance imaging features. Mult Scler 19(1):76-86. https://doi.org/10.1177/1352458512445944

3. Bhatt P, Bray L, Raju S, Dapaah-Siakwan F, Patel A, Chaudhari R et al (2019) Temporal trends of pediatric hospitalizations with acute disseminated encephalomyelitis in the United States: An Analysis from 2006 to 2014 using National Inpatient Sample. J Pediatr 206: 26-32.e1. https://doi.org/10.1016/j.jpeds.2018.10.044

4. Abdi S, Ghorbani A, Fatehi F (2020) The association of SARSCoV-2 infection and acute disseminated encephalomyelitis without prominent clinical pulmonary symptoms. J Neurol Sci 416:117001. https://doi.org/10.1016/j.jns.2020.117001

5. Yong MH, Chan YFZ, Liu J, Sanamandra SK, Kheok SW, Lim KC, Sewa DW (2020) A Rare Case of Acute Hemorrhagic Leukoencephalitis in a COVID-19 Patient. J Neurol Sci 416: 117035. https://doi.org/10.1016/j.jns.2020.117035

6. Chalil A, Baker C, Johnston R, Just C, Debicki D, Mayich et al (2020) Acute hemorrhagic encephalitis related to COVID-19. Neurol Clin Pract:10.1212/CPJ.0000000000000900

7. Handa R, Nanda S, Prasad A, Anand R, Zutshi D, Dass SK, Bedi PK, Pahuja A, Shah PK, Sharma B (2020) Covid-19-associated acute haemorrhagic leukoencephalomyelitis. Neurol Sci 41(11): 3023-3026. https://doi.org/10.1007/s10072-020-04703-z

8. Karapanayiotides T, Geka E, Prassopoulos P, Koutroulou I, Kollaras P, Kiourtzieva E et al (2020) Concentric demyelination pattern in COVID-19-associated acute haemorrhagic leukoencephalitis: a lurking catastrophe? Brain 143(12):e100. https://doi.org/10.1093/brain/awaa375

9. Perrin P, Collongues N, Baloglu S, Bedo D, Bassand X, Lavaux T, Gautier-Vargas G, Keller N, Kremer S, Fafi-Kremer S, Moulin B, Benotmane I, Caillard S (2021) Cytokine release syndromeassociated encephalopathy in patients with COVID-19. Eur J Neurol 28(1):248-258. https://doi.org/10.1111/ene.14491

10. Ghosh R, Dubey S, Finsterer J, Chatterjee S, Ray BK (2020) SARS-CoV-2-associated acute hemorrhagic, necrotizing encephalitis (ahne) presenting with cognitive impairment in a 44-year-old woman without comorbidities: a case report. Am J Case Rep 21: e925641. https://doi.org/10.12659/AJCR.925641

11. Haqiqi A, Samuels TL, Lamb FJ, Moharrum T, Myers AE (2021) Acute haemorrhagic leukoencephalitis (Hurst Disease) in severe COVID-19 infection. Brain Behav Immun Health, 100208. https://doi.org/10.1016/j.bbih.2021.100208

12. Waak M, Malone S, Sinclair K, Phillips G, Bandodkar S, Wienholt L, Robertson T, Whitehead B, Trnka P, Kothur K, Dale RC (2019) Acute hemorrhagic leukoencephalopathy: pathological features and cerebrospinal fluid cytokine profiles. Pediatr Neurol 100:92-96. https://doi.org/10.1016/j.pediatrneurol.2019.06.013

13. Hanafi R, Roger PA, Perin B, Kuchcinski G, Deleval N, Dallery F, Michel D, Hacein-Bey L, Pruvo JP, Outteryck O, Constans JM (2020) COVID-19 Neurologic Complication with CNS Vasculitis-Like Pattern. AJNR Am J Neuroradiol 41(8):13841387. https://doi.org/10.3174/ajnr.A6651

14. Lann MA, Lovell MA, Kleinschmidt-DeMasters BK (2010) Acute hemorrhagic leukoencephalitis: a critical entity for forensic pathologists to recognize. Am J Forensic Med Pathol 31(1):7-11. https:// doi.org/10.1097/PAF.0b013e3181c6be92

15. von Weyhern CH, Kaufmann I, Neff F, Kremer M (2020) Early evidence of pronounced brain involvement in fatal COVID-19 outcomes. Lancet. 395(10241):e109. https://doi.org/10.1016/ S0140-6736(20)31282-4

16. Reichard RR, Kashani KB, Boire NA, Constantopoulos E, Guo Y, Lucchinetti CF (2020) Neuropathology of COVID-19: a spectrum of vascular and acute disseminated encephalomyelitis (ADEM)-like pathology. Acta Neuropathol 140(1):1-6. https://doi.org/10.1007/ s00401-020-02166-2

17. Al-Sarraj S, Troakes C, Hanley B, Osborn M, Richardson MP, Hotopf M et al (2020) Invited review: the spectrum of neuropathology in COVID-19. Neuropathol Appl Neurobiol. https://doi.org/10. 1111/nan.12667

18. Kremer S, Lersy F, de Sèze J, Ferré JC, Maamar A, Carsin-Nicol B, Collange O, Bonneville F, Adam G, Martin-Blondel G, Rafiq M, Geeraerts T, Delamarre L, Grand S, Krainik A, For the SFNRCOVID Group, Kremer S, Adam G, Alleg M, Anheim M, Anxionnat R, Ardellier FD, Baloglu S, Bapst B, Benzakoun J, Berge J, Bolognini F, Bonneville F, Bornet G, Boulay C, Boulouis G, Boutet C, Brisset JC, Caillard S, Carré S, CarsinNicol B, Collange O, Comby PO, Constans JM, David JS, de Beaurepaire I, de Sèze J, Delamarre L, Desal H, Edjlali-Goujon M, Fabre X, Fafi-Kremer S, Ferré JC, Feuerstein P, Henry Feugeas MC, Forestier G, Gaudemer A, Geeraerts T, Grand S, Hansmann Y, Heintz A, Helms J, Hemmert C, Hmeydia G, Jager L, Kazémi A, Kerleroux B, Khalil A, Krainik A, Lacalm A, Lecler A, Lecocq C, Lefèbvre N, Lersy F, Maamar A, Martin-Blondel G, Matthieu M, Megdiche I, Mertes PM, Messié J, Metanbou S, Meyer N, Meziani F, Mutschler V, Nesser P, Oesterlé H, Ohana M, Oppenheim C, Pyatigorskaya N, Rafiq M, Ricolfi F, Saleme S, Schenck M, Schmitt E, Schneider F, Sebag N, Talla Mba Y, Thouant P, Willaume T, Zhu F, Zorn PE, Cotton F (2020) Brain MRI findings in severe COVID-19: a retrospective observational study. Radiology 297(2):E242-E251. https://doi.org/10.1148/ radiol.2020202222

19. Agarwal S, Jain R, Dogra S, Krieger P, Lewis A, Nguyen V, Melmed K, Galetta S (2020) Cerebral microbleeds and leukoencephalopathy in critically ill patients with COVID-19. Stroke 51(9):2649-2655. https://doi.org/10.1161/STROKEAHA. 120.030940

20. Dixon L, Varley J, Gontsarova A, Mallon D, Tona F, Muir D et al (2020) COVID-19-related acute necrotizing encephalopathy with brain stem involvement in a patient with aplastic anemia. Neurol Neuroimmunol Neuroinflamm 7(5):e789. https://doi.org/10.1212/ NXI.0000000000000789

21. Paterson RW, Brown RL, Benjamin L, Nortley R, Wiethoff S, Bharucha $\mathrm{T}$ et al (2020) The emerging spectrum of COVID-19 neurology: clinical, radiological and laboratory findings. Brain 143(10):3104-3120. https://doi.org/10.1093/brain/awaa240

22. Arenas RD, Hernandez ES (2020) Pseudotumoral lesion as a manifestation of acute hemorrhagic leukoencephalitis (Weston-Hurst syndrome). Mult Scler Relat Disord 46:10258.3. https://doi.org/ 10.1016/j.msard.2020.102583

23. Abou Zeid NE, Burns JD, Wijdicks EF, Giannini C, Keegan BM (2010) Atypical acute hemorrhagic leukoencephalitis (Hurst's disease) presenting with focal hemorrhagic brainstem lesion. Neurocrit Care 12(1):95-97. https://doi.org/10.1007/s12028-009-9293-x

24. Radmanesh A, Derman A, Lui YW, Raz E, Loh JP, Hagiwara M, Borja MJ, Zan E, Fatterpekar GM (2020) COVID-19-associated diffuse leukoencephalopathy and microhemorrhages. Radiology 297(1):E223-E227. https://doi.org/10.1148/radiol.2020202040

25. Kuperan S, Ostrow P, Landi MK, Bakshi R (2003) Acute hemorrhagic leukoencephalitis vs ADEM: FLAIR MRI and neuropathology findings. Neurology 60(4):721-722. https://doi.org/10.1212/ 01.wnl.0000048493.82053.4c

26. Poyiadji N, Shahin G, Noujaim D, Stone M, Patel S, Griffith B (2020) COVID-19-associated acute hemorrhagic necrotizing 
encephalopathy: imaging features. Radiology 296(2):E119-E120. https://doi.org/10.1148/radiol.2020201187

27. Morvan A-C, Kerambrun H (2020) Fatal necrotizing encephalitis associated with COVID-19: a case report. Neurol Clin Pract 10: 1212. https://doi.org/10.1212/CPJ.0000000000000945

28. Virhammar J, Kumlien E, Fällmar D, Frithiof R, Jackmann S, Sköld MK, Kadir M, Frick J, Lindeberg J, Olivero-Reinius H, Ryttlefors M, Cunningham JL, Wikström J, Grabowska A, Bondeson K, Bergquist J, Zetterberg H, Rostami E (2020) Acute necrotizing encephalopathy with SARS-CoV-2 RNA confirmed in cerebrospinal fluid. Neurology 95(10):445-449. https://doi.org/10.1212/ WNL.0000000000010250

29. Cavalcanti DD, Raz E, Shapiro M, Dehkharghani S, Yaghi S, Lillemoe K et al (2020) Cerebral venous thrombosis associated with COVID-19. AJNR Am J Neuroradiol 41(8):1370-1376. https://doi.org/10.3174/ajnr.A6644

30. Parauda SC, Gao V, Gewirtz AN, Parikh NS, Merkler AE, Lantos J et al (2020) Posterior reversible encephalopathy syndrome in patients with COVID-19. J Neurol Sci 416:117019. https://doi. org/10.1016/j.jns.2020.117019

31. Rosa Júnior M, Borges ÉI, Fonseca APA, Fiorot JL, Balarini L, Valim V (2018) Posterior reversible encephalopathy syndrome during treatment with tocilizumab in juvenile idiopathic arthritis. Arq Neuropsiquiatr 76(10):720-721. https://doi.org/10.1590/0004282X20180093

32. Princiotta Cariddi L, Tabaee Damavandi P, Carimati F, Banfi P, Clemenzi A, Marelli M, Giorgianni A, Vinacci G, Mauri M, Versino M (2020) Reversible encephalopathy syndrome (PRES) in a COVID-19 patient. J Neurol 267(11):3157-3160. https://doi. org/10.1007/s00415-020-10001-7

Publisher's note Springer Nature remains neutral with regard to jurisdictional claims in published maps and institutional affiliations. 